\title{
Direct Measurement of Isotope Ratio of Antimony in Seawater by Hydride Generation/Inductively Coupled Plasma Mass Spectrometry
}

\author{
Takahiro KumamarU*十, Manabu Yamamoto*, Fumino NaKaTA*, \\ Hiroyuki Tsubota** and Ko-ichi NishiKIDA*** \\ *Department of Chemistry, Faculty of Science, Hiroshima University, \\ Kagamiyama, Higashi-Hiroshima 724, Japan \\ **Division of Environmental Science, Faculty of Integrated Arts and Sciences, Hiroshima University, \\ Kagamiyama, Higashi-Hiroshima 730, Japan \\ *** Perkin-Elmer Corporation, Organic Analysis Division, Main Ave., Norwalk, CT 06859, USA
}

Keywords Isotope ratio, antimony, seawater, hydride generation, stibine, inductively coupled plasma mass spectrometry

Hydride generation (HG) is one of the most reliable separation and condensation techniques for the hydrideforming elements in aqueous matrices. In our previous study', flow injection (FI)/HG-atomic absorption spectrometry (AAS) was developed to treat a large number of samples and to analyze trace levels of arsenic, antimony, bismuth, selenium and tellurium. The FI/ HG technique was also applied to inductively coupled plasma atomic emission spectrometry (ICP-AES). ${ }^{2}$ Since in the FI/HG system the analytes were introduced into the analytical zone as gaseous species, the sensitivities of AAS and ICP-AES were improved by 1 to 2 orders-of-magnitude when the FI/HG technique instead of a conventional solution nebulization method was coupled with AAS or ICP-AES. Recently, ICP mass spectrometry (ICP-MS) has been used successfully in the field of ultra-trace analysis. ${ }^{3-5}$ Although ICP-MS with solution nebulization is generally much more sensitive than AAS and ICP-AES and moreover is capable of measuring isotope ratios, the method has been shown to be susceptible to all complex matrices accompanying the analytes. In such cases, the HG procedure is considered to be the most favorable pretreatment process for the determination of hydrideforming elements in trace levels by ICP-MS, because the HG technique not only enables us to remove the matrix effect, but also has a potential to improve their detection limits, for the same reason as mentioned above for HG-AAS and HG-ICP-AES.

Powell et al. optimized the operating conditions for HG-ICP-MS and showed that it actually improved detection limits of arsenic, selenium, mercury, antimony,

† To whom correspondence should be addressed. bismuth and tellurium by 1 to 2 orders-of-magnitude compared with those of solution-nebulization ICP-MS. ${ }^{6}$ As for seawater analysis by ICP-MS, McLaren et al. ${ }^{7}$ analyzed trace metal elements in coastal seawater and open ocean-water reference materials by the isotope dilution method. They first removed major constituents such as alkali and alkaline earth salts and then concentrated the analytes by column chromatography with an 8-hydroxyquinoline-immobilized silica-gel column.

In this paper, the authors demonstrate an FI/HG-ICPMS for the direct measurement of antimony isotopes, ${ }^{121} \mathrm{Sb}$ and ${ }^{123} \mathrm{Sb}$ in seawater; these are the two naturally occurring stable antimony isotopes.

\section{Experimental}

\section{Apparatus}

The manifold for the continuous HG and its interfacing to an ICP-mass spectrometer are depicted in Fig. 1. The construction of the HG system is nearly the same as described in the previous paper. ${ }^{8}$ Since the continuous operation mode was employed in this work, the same tubing unit as that in the previous study ${ }^{2}$ was utilized without the injection valve for sample introduction. A pump-drained liquid-gas separator was used instead of a conventional non-pumping separator ${ }^{9}$ or membrane tube separator ${ }^{8}$, because the latter two separation techniques gave degraded reproducibilities.

A Perkin-Elmer Sciex Model Elan 500 ICP-mass spectrometer equipped with a water-cooled spray chamber and a Meinhardt concentric nebulizer was used. 


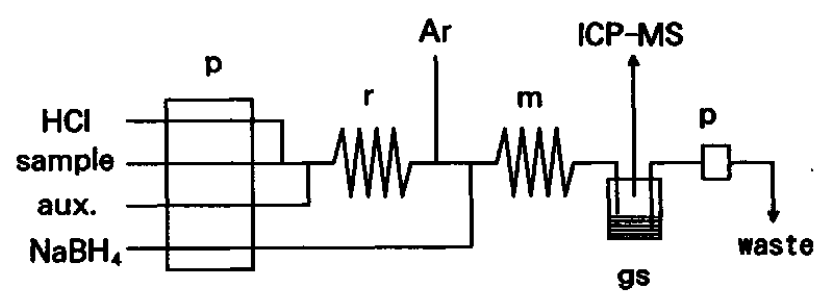

Fig. 1 A continuous hydride generation manifold: (p) peristaltic pump; (r) pre-reduction coil; (m) mixing coil; (gs) gas-liquid separator.

Table 1 Operating conditions

\begin{tabular}{|c|c|}
\hline \multicolumn{2}{|c|}{ Continuous hydride generator } \\
\hline $\mathrm{HCl}$ & $1.0 \mathrm{M} ; 7 \mathrm{ml} / \mathrm{min}$ \\
\hline $\mathrm{NaBH}_{4}$ & $3 \%$ in $0.25 \% \mathrm{NaOH} ; 1.5 \mathrm{ml} / \mathrm{min}$ \\
\hline Aux. & $\mathrm{KI}, 50 \% ; 1.5 \mathrm{ml} / \mathrm{min}$ \\
\hline Sample & $\mathrm{HCl}$ acidity, $1.0 \mathrm{M} ; 7.0 \mathrm{ml} / \mathrm{min}$ \\
\hline Pre-reduction coil & $3 \mathrm{~mm}$ i.d., $1 \mathrm{~m}$ long \\
\hline Mixing coil & $3 \mathrm{~mm}$ i.d., $20 \mathrm{~cm}$ long \\
\hline Separator & pump-drained type \\
\hline Carrier gas & $\mathrm{Ar}, 0.11 / \mathrm{min}$ \\
\hline \multicolumn{2}{|c|}{ ICP r.f. generator (Perkin-Elmer Sciex Elan 500) } \\
\hline Plasma r.f. power & $1.2 \mathrm{~kW}$ \\
\hline Plasma gas & Ar, $12.01 / \mathrm{min}$ \\
\hline Aux. gas & Ar, $1.01 / \mathrm{min}$ \\
\hline Injector gas & $\mathrm{Ar}, 0.51 / \mathrm{min}$ \\
\hline \multicolumn{2}{|c|}{ Mass spectrometer (Perkin-Elmer Sciex Elan 500) } \\
\hline Mode selection & isotope ratio mode \\
\hline Resolution & low $(1.0 \mathrm{amu})$ \\
\hline Measurements & 1 peak \\
\hline Measurement mode & multi (peak hopping) \\
\hline Integration time & $1.0 \mathrm{~s}$ \\
\hline Dwell time & variable (changed for sample type) \\
\hline
\end{tabular}

The temperature of the spray chamber was kept at 2 to $3^{\circ} \mathrm{C}$ and stibine was introduced into the torch through the nebulizer.

\section{Reagents}

Both nitric acid and hydrochloric acid were ultrapuregrade reagents which contained metal elements at concentrations lower than $1 \mathrm{pg} / \mathrm{l}$. Antimony stock solutions were prepared by diluting a commercially available standard solution of antimony $(1000 \mathrm{mg} / \mathrm{l}$, Kanto Chemicals, Tokyo, Japan) with $0.1 \mathrm{M}$ nitric acid. Other reagents such as sodium borohydride and potassium iodide were of analytical reagent grade. The use of any reagent which caused the background count to exceed several hundreds per second was avoided.

\section{Operating conditions}

The recommended operating conditions to measure the isotope ratio and the detection limit are given in Table 1 .

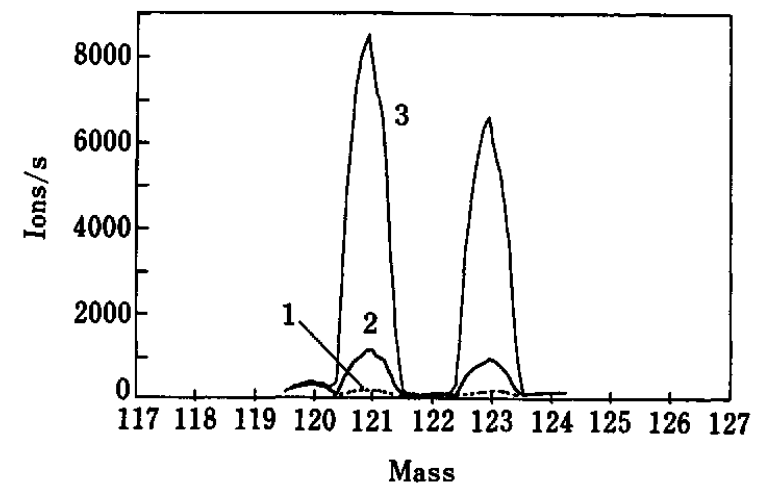

Fig. 2 Mass spectra of Sb in seawater: (1) reagent blank; (2) seawater [Station M-7 (To-83-18), $18^{\circ} 13.22^{\prime} \mathrm{N}, 144^{\circ} 42.07^{\prime} \mathrm{W}$; (3) seawater spiked with $1 \mu \mathrm{gSb} / 1$.

\section{Results and Discussion}

\section{Interference}

Prior to the isotope-ratio determination, a semiquantitative analysis was carried out for spiked $(1.0 \mu \mathrm{g}$ $\mathrm{Sb} / \mathrm{l}$ ) and unspiked seawater by the solution nebulization and the HG methods in order to survey the overall mass spectra. For the nebulization method, the sample was diluted with water to reduce its salinity. A typical example of the spectra obtained by the HG method is shown in Fig. 2. As expected, the HG method enhanced the ion intensities of the antimony isotopes nearly 2 orders-of-magnitude compared with the nebulization method. Besides, the hydride introduction system is essentially free from water, and therefore largely suppressed the levels of metal oxides which appeared in the nebulization of the aqueous solution, resulting in far simpler mass spectra. Hence, there were fewer chances of isobaric and spectral'interferences in the HG-ICP-MS.

Under the same experimental conditions for the stibine generation, arsenic, tellurium, germanium and bismuth also generate their hydrides. Among these elements, the isobaric interference of ${ }^{123} \mathrm{Te}$ on ${ }^{123} \mathrm{Sb}$ might be observed for seawater. However, as the level of tellurium is quite low and moreover the natural isotope abundance of ${ }^{123} \mathrm{Te}$ is only $0.9 \%$, it is obvious that such isobaric interference is improbable in this work.

\section{Detection limit}

The detection limit, defined as twice the standard deviation of the background, was $6.0 \mathrm{ngSb} / 1$ by the proposed HG-ICP-MS. Therefore, this method is sensitive enough to measure the concentration of antimony in seawater without any preconcentration.

\section{Memory effect}

Throughout this work, a memory effect for antimony was observed in prolonged experiments. Since the effect was removed by the replacement of both sampler and skimmer cones, it could be caused by the formation of Sb-Ni alloy on the surface of nickel cones. 

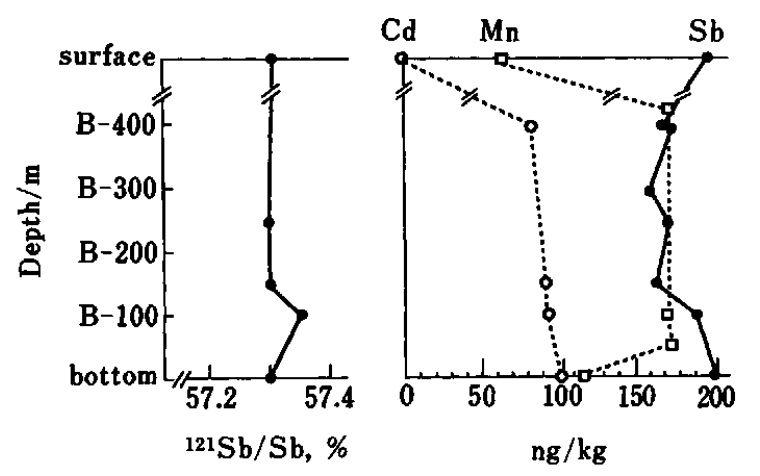

Fig. 3 Vertical distributions of ${ }^{121} \mathrm{Sb} / \mathrm{Sb}, \mathrm{Sb}, \mathrm{Cd}$ and $\mathrm{Mn}$ in seawater. Station M-7 (To-83-18), $18^{\circ} 13.22^{\prime} \mathrm{N}, 144^{\circ} 42.07^{\prime} \mathrm{W}$; bottom depth, $3730 \mathrm{~m}$; $\mathrm{Cd}$ and $\mathrm{Mn}$ were determined by graphite furnace atomic absorption spectrometry.

\section{Measurement of isotope ratio}

For the antimony isotope-ratio measurements, the solutions containing $0.4,1.0$ and $10.0 \mu \mathrm{g} / \mathrm{l}$ of antimony and a blank solution were measured. The observed isotope ratio, ${ }^{121} \mathrm{Sb} / \mathrm{Sb}$ (where $\mathrm{Sb}={ }^{121} \mathrm{Sb}+{ }^{123} \mathrm{Sb}$ ), first increased and then leveled off at a constant value of $56.8 \pm 0.01 \%$ as the ion intensity of ${ }^{121} \mathrm{Sb}$ increased. The isotope ratio reported in the literature ${ }^{10}$ is $57.4 \%$. Consequently, the mass discrimination factor was calculated to be $1.01 \pm 0.01$ for ${ }^{121} \mathrm{Sb} / \mathrm{Sb}$, when the ion intensity of ${ }^{121} \mathrm{Sb}$ exceeded $c a .5 \times 10^{3}$ counts, independent of the antimony concentration or integration time.

\section{Seawater analysis}

The seawater samples analyzed in this work were collected during cruise To-83-18 of Tokai Daigaku Maru II at Station M-7 $\left(18^{\circ} 13.22^{\prime} \mathrm{N}, 144^{\circ} 42.07^{\prime} \mathrm{W}\right.$; $3730 \mathrm{~m}$ deep) in the Mariana trench in the Pacific Ocean by using clean samplers developed by Tsubota $e t$ al. ${ }^{11}$ The depth profiles of antimony concentration, together with those of cadmium and manganese and the isotope ratio $\left({ }^{121} \mathrm{Sb} / \mathrm{Sb}\right)$, are given in Fig. 3. The antimony concentration was measured by a calibration method.
The calibration curve was linear up to $0.5 \mu \mathrm{gSb} / \mathrm{l}$ for both ${ }^{121} \mathrm{Sb}$ and ${ }^{123} \mathrm{Sb}$. The isotope ratios shown in Fig. 3 were corrected by the mass discrimination factor. From a separate study, distinctly high metal concentrations were observed for heavy metal ions such as silver, lead and copper in near bottom water, i.e., 50 to $100 \mathrm{~m}$ above the bottom, which might be due to the presence of hydrothermal reaction in this area. ${ }^{12}$ A deviation of the antimony isotope ratio observed just above the bottom may indicate a certain effect of such a reaction. Detailed discussions on this phenomenon will be reported elsewhere.

The authors wish to thank the late Prof. M. Murozumi of Muroran Institute of Technology for his valuable suggestions.

\section{References}

1. M. Yamamoto, M. Yasuda and Y. Yamamoto, Anal. Chem., 57, 1382 (1985).

2. F. Nakata, H. Sunahata, H. Fujimoto, M. Yamamoto and T. Kumamaru, J. Anal. At. Spectrom., 3, 579 (1988).

3. A. L. Gray, Spectrochim. Acta, 40B, 1525 (1985).

4. R. S. Houk, Anal. Chem., 58, 97A (1986).

5. H. Kawaguchi, Anal. Sci., 4, 339 (1988).

6. M. J. Powel, D. W. Boomer and R. J. McVicars, Anal. Chem., 58, 2864 (1986).

7. J. W. McLaren, A. P. Mykytiuk, S. N. Willie and S. S. Berman, Anal. Chem., 57, 2907 (1985).

8. M. Yamamoto, T. Takada, T. Kumamaru, Y. Yasuda, Y. Yokoyama and Y. Yamamoto, Anal. Chem., 59, 2446 (1987).

9. P. N. Vijan, A. C. Rayner, D. Sturgis and G. R. Wood, Anal. Chim. Acta, 82, 329 (1976).

10. "Handbook of Chemistry and Physics", ed. D. R. Lide, 73rd ed., CRC Press, Boca Raton, 1992.

11. H. Tsubota, in "The Distribution and Behavior of Heavy Metals in the Ocean", ed. K. Kajiura, p. 11, Koseisha Koseikaku, Tokyo, 1985.

12. M. Morozumi, in "The Distribution and Behavior of Heavy Metals in the Ocean", ed. K. Kajiura, p. 273, Koseisha Koseikaku, Tokyo, 1985. 